\title{
MEGARA. Large pupil element tests and performance
}

\author{
I., Martínez-Delgado ${ }^{a}$, E., Sánchez-Blanco ${ }^{\mathrm{a}}$, A., Pérez-Calpena ${ }^{\mathrm{a}}$, M.L., García-Vargas ${ }^{\mathrm{a}}$, X., M. \\ Maldonado ${ }^{\mathrm{a}}$, A., Gil de Paz ${ }^{\mathrm{b}}$, E., Carrasco ${ }^{\mathrm{c}}$, J., Gallego ${ }^{\mathrm{b}}$, J., Iglesias-Páramo ${ }^{\mathrm{d}}$, F.M., Sánchez- \\ Moreno $^{\mathrm{e}}$, \& MEGARA Team \\ ${ }^{a}$ FRACTAL SLNE, C/ Tulipán 2, Portal 13 1A, E-28231, Las Rozas de Madrid, Spain; \\ ${ }^{\mathrm{b}}$ GUAIX Group, Astrophysics Department, Universidad Complutense de Madrid, Av. Complutense \\ s/n, E-28040, Madrid, Spain; \\ 'Instituto Nacional de Astrofísica, Óptica y Electrónica, Luis Enrique Erro \#1, Tonanzintla, Puebla, \\ 72840, Mexico; \\ ${ }^{d}$ Instituto de Astrofísica de Andalucía, Glorieta de Astronomía s/n, E-18008, Granada, Spain; \\ ${ }^{e}$ Facultad de Informática, Universidad Politécnica de Madrid, Campus de Montegancedo, E-28660, \\ Boadilla del Monte, Madrid, Spain;
}

\begin{abstract}
MEGARA is a third generation spectrograph for the Spanish $10.4 \mathrm{~m}$ telescope (GTC) providing two observing modes: a large central Integral Field Unit (IFU), called the Large Compact Bundle (LCB), covering a FOV of $12.5 \times 11.3 \mathrm{arcsec}^{2}$, and a Multi-Object Spectrograph (MOS) with a FOV of $3.5 \times 3.5 \mathrm{arcmin}^{2}$. MEGARA will observe the whole visible range from $3650 \mathrm{~A}$ to $10000 \mathrm{~A}$ allowing different spectral resolutions (low, medium and high) with $\mathrm{R}=6000,11000$ and 18000 respectively. The dispersive elements are placed at the spectrograph pupil position in the path of the collimated beam and they are composed of a set of volume phase hologram gratings (VPHs) sandwiched between two flat windows and coupled in addition to two prisms in the case of the medium- and high-resolution units. We will describe the tests and setups developed to check the requirements of all units, as well as the obtained performance at laboratory
\end{abstract}

Keywords: MEGARA, integral field unit (IFU), multi-object spectrograoh (MOS), VPH, gratings, holographic gratings, GTC

\section{INTRODUCTION}

MEGARA is a third generation instrument for the Spanish $10.4 \mathrm{~m}$ telescope (GTC). It is an optical Integral Field Unit (IFU) and Multi-Object Spectrograph (MOS). The spectrograph will be fiber-fed from the GTC Folded-Cassegrain station to the Nasmyth platform, through a collection of fibers coupled to microlens arrays arranged in an IFU, known as Large Compact Bundle (LCB), and a Multi-Object Spectrograph (MOS) mode that is configured with a collection of 100 robotic positioners with a 7-fiber mini-bundle each. The fibers layout is shown in Figure 1. Finally, each fiber bundle configuration (LCB and MOS) shall define a pseudo-slit at the instrument entrance delivering the same spaxel scale $\left(\sim 0.62^{\prime \prime}\right)$ on the sky (fiber core is $100 \mu \mathrm{m}$ for both modes), and a plate scale at the detector focal plane of $0.186^{\prime \prime}$ per pixel (equivalent to 4-pixels resolution).

Light is coming from the telescope with an $\mathrm{f} / 17$ focal ratio that will be converted into a faster beam (f/3), for minimizing the impact of the FRD before the beam comes into the fiber core, thanks to a set of microlens arrays. In order to let all the microlenses to see the same pupil, the light beam coming through the focal plane will be driven though a field lens that will allow illuminating the lens-arrays with a telecentric ray beam. That is, the axes of all the fiber bundles are parallel among them. 


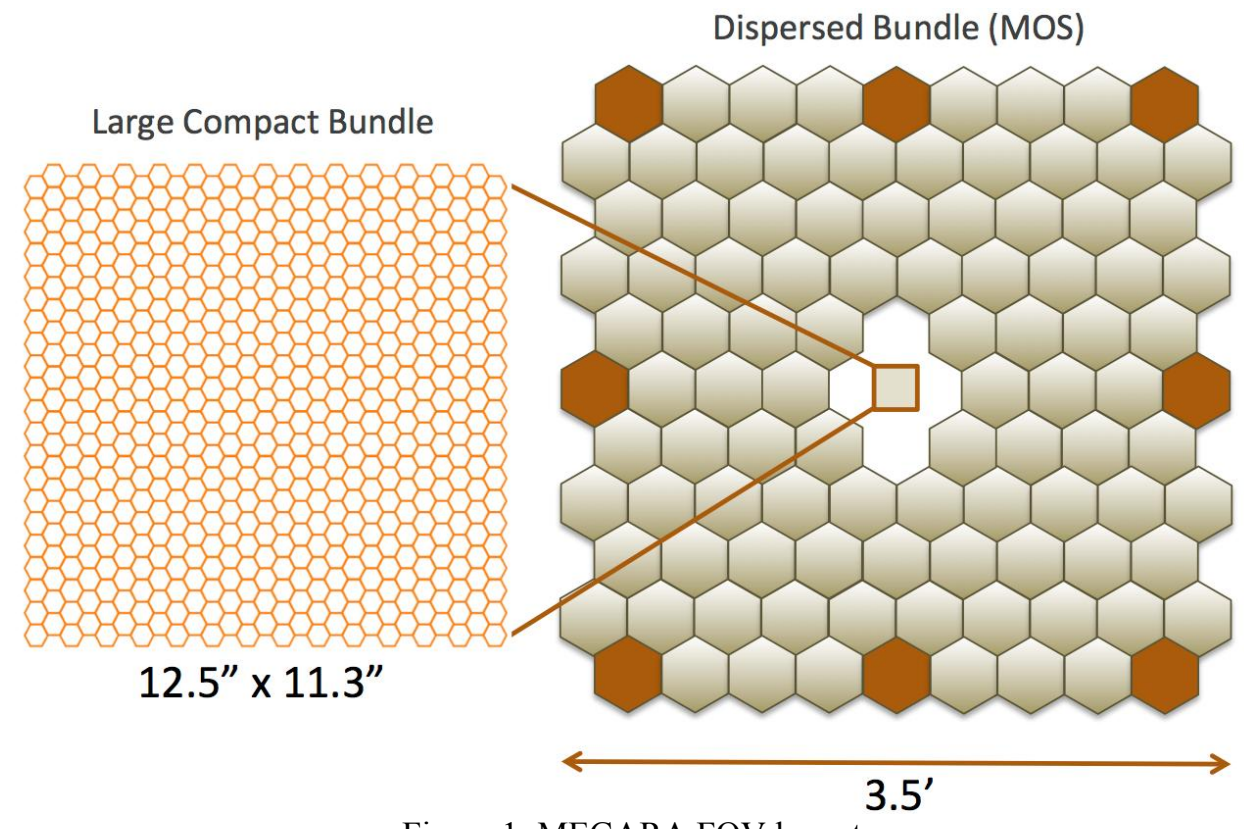

Figure 1: MEGARA FOV layout

The spectrograph is equipped with a total of 18 dispersive elements placed at the pupil position in the path of the collimated beam, although the grating's wheel is designed to load 11 in each observation run (see Figure 2). The whole set offers three resolutions modes, low (LR), medium (MR) and high (HR) providing the final spectrograph with power resolutions of $\sim 6000,12000$, and 19000 respectively, see Figure 3. The AOI to each pupil element is found to be 34 degrees.

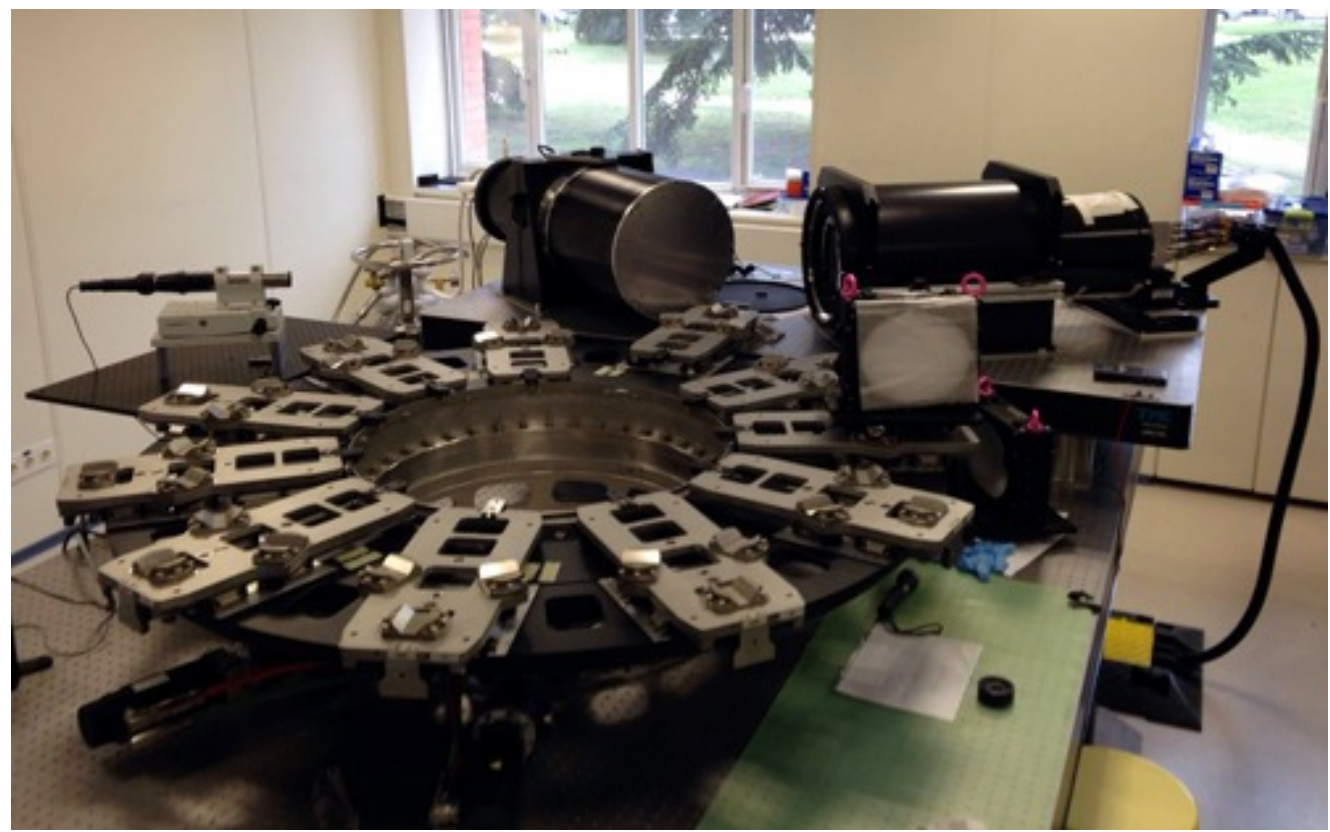

Figure 2: Overview of MEGARA during AIV and alignment process 


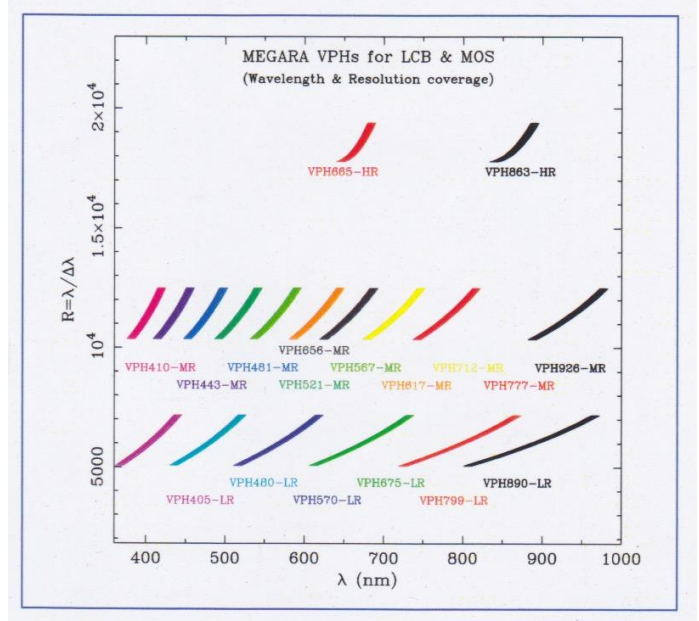

Figure 3: The power resolution given by each MEGARA's grating

The 18 gratings are split into $6 \mathrm{LR}, 10 \mathrm{MR}$ and $2 \mathrm{HR}$. All of them are volume phase holograms sandwiched between two flat windows with AR coatings, although in the case of the medium- and high-resolution configurations, the VPHs are sandwiched between two PBM2Y prisms joined by means of RTV-141 silicone. In these last cases, the AR coatings are applied to the external faces of each prism. The flat widows and prisms (manufacturing and polishing) and AR coatings treatments have been performed by INAOE, while Wasatch Photonics manufactured the phase holograms.

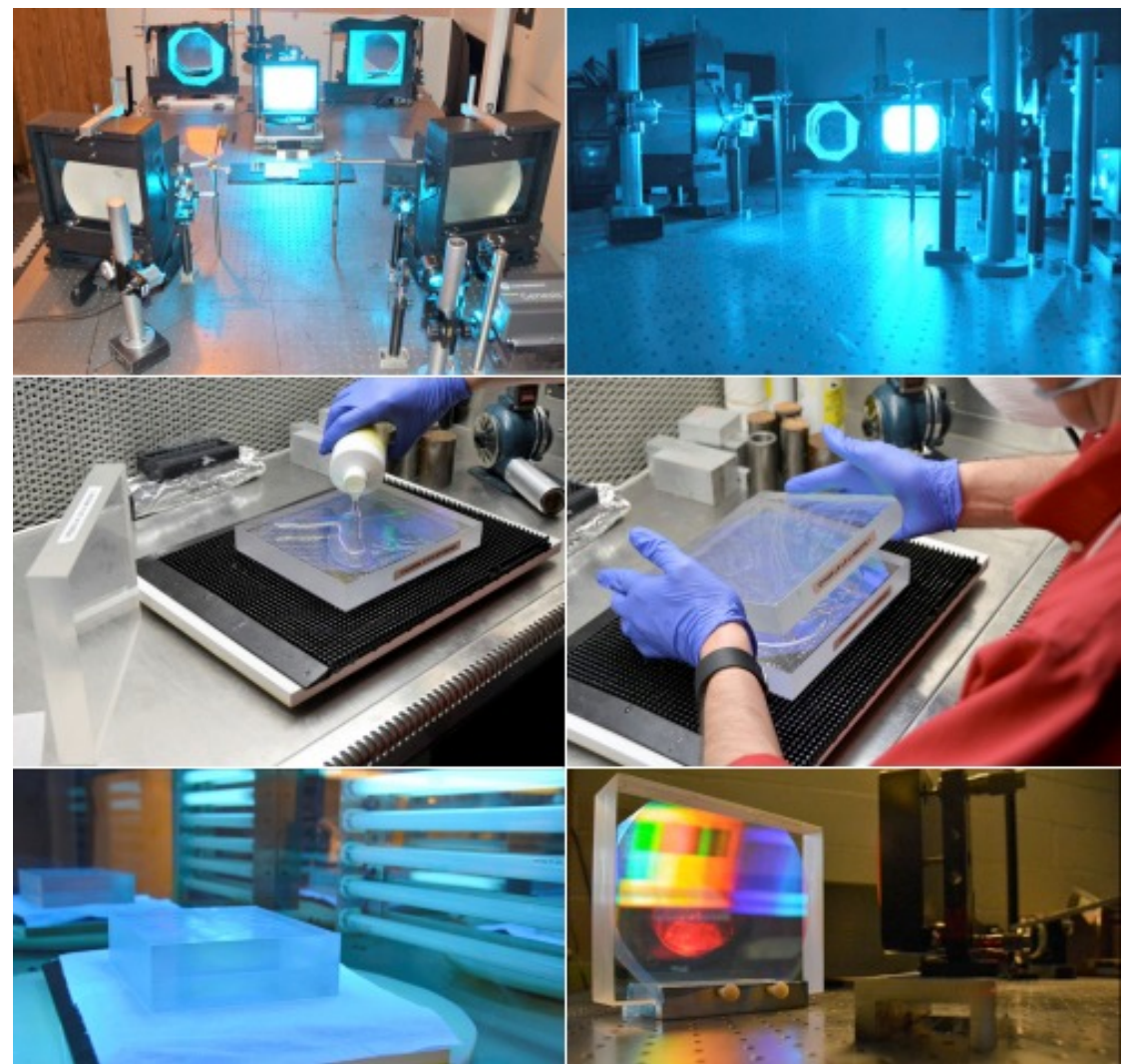

Figure 4: MEGARA Phase holograms manufacturing process at Wasatch facilities 
The main physical parameters of each VPH can be found in Table 1, Figure 5, Figure 6 and Figure 7 respectively. In the case of MR and HR prisms, the physical dimensions an/or angles are shown in Figure 8 and Figure 9. The main optical parameters of each grating are found in Table 2.

Table 1: Dimensional specifications of each grating type

\begin{tabular}{|c|c|c|c|c|}
\cline { 2 - 5 } \multicolumn{1}{c|}{} & \multicolumn{4}{c|}{ Physical Parameters } \\
\hline VPH & Length (mm) & Height (mm) & Width (mm) & Weight (g) \\
\hline LR & $180.0 \pm 0.2$ & $220.0 \pm 0.2$ & $60.0 \pm 0.2$ & $5230 \pm 29$ \\
\hline MR & $190.0 \pm 0.2$ & $240.0 \pm 0.2$ & $40.0 \pm 0.2$ & $4015 \pm 29$ \\
\hline HR & $190.0 \pm 0.2$ & $240.0 \pm 0.2$ & $40.0 \pm 0.2$ & $4015 \pm 29$ \\
\hline
\end{tabular}
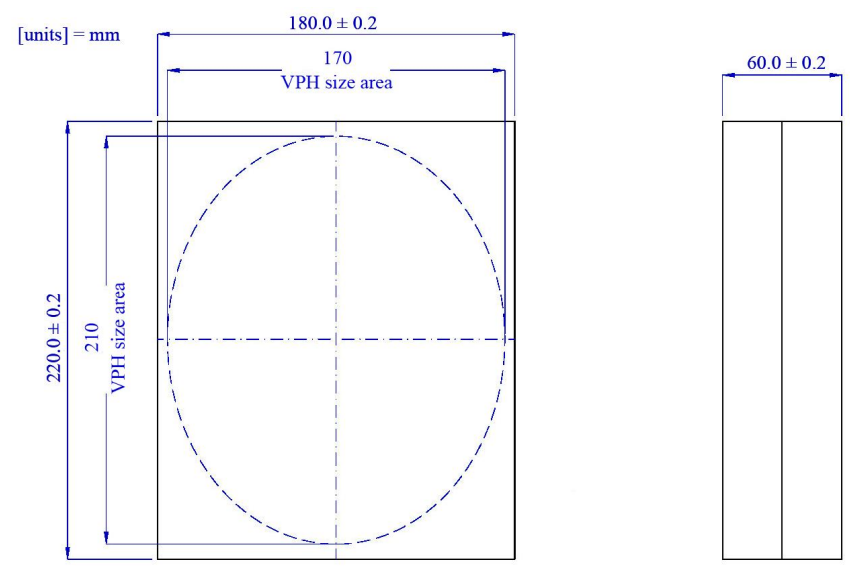

Figure 5: LR-VPH drawing map where physical dimensions are shown
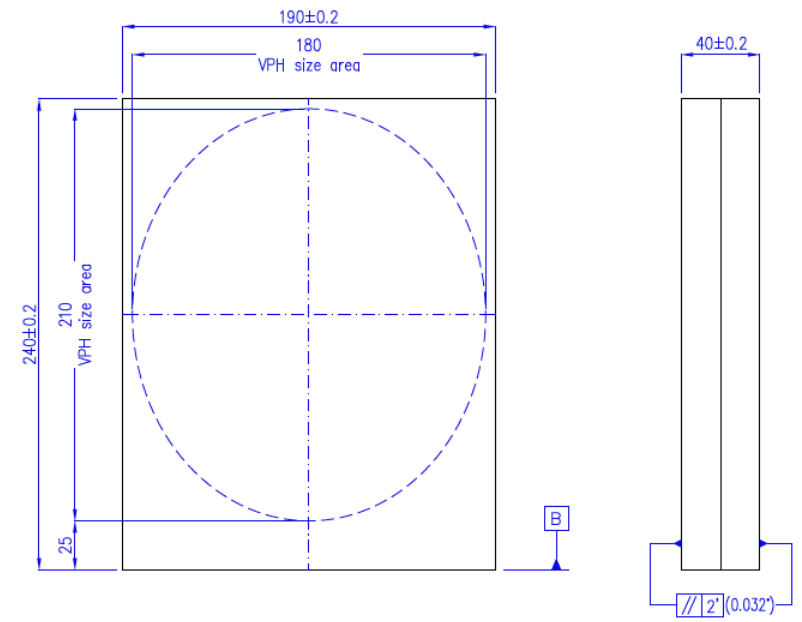

Figure 6: MR-VPH drawing map where physical dimensions are shown 


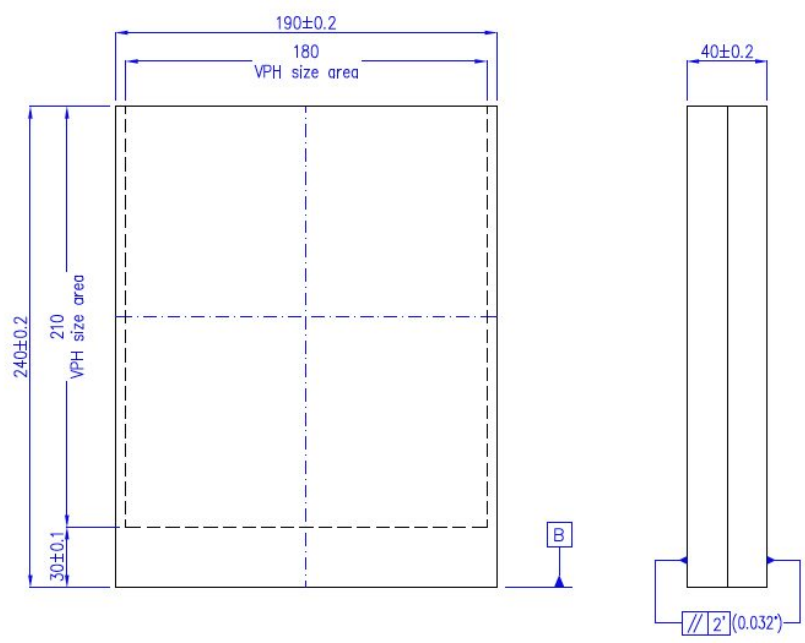

Figure 7: HR-VPH drawing map where physical dimensions are shown
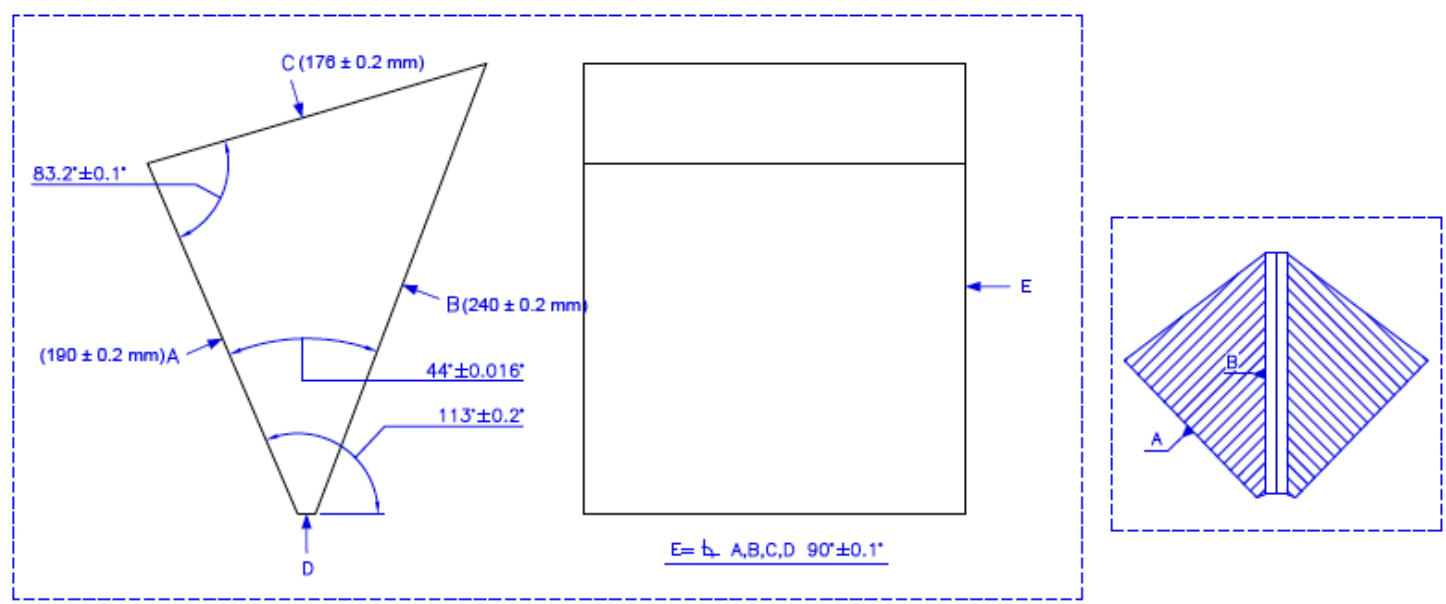

Figure 8: MR prisms drawing map
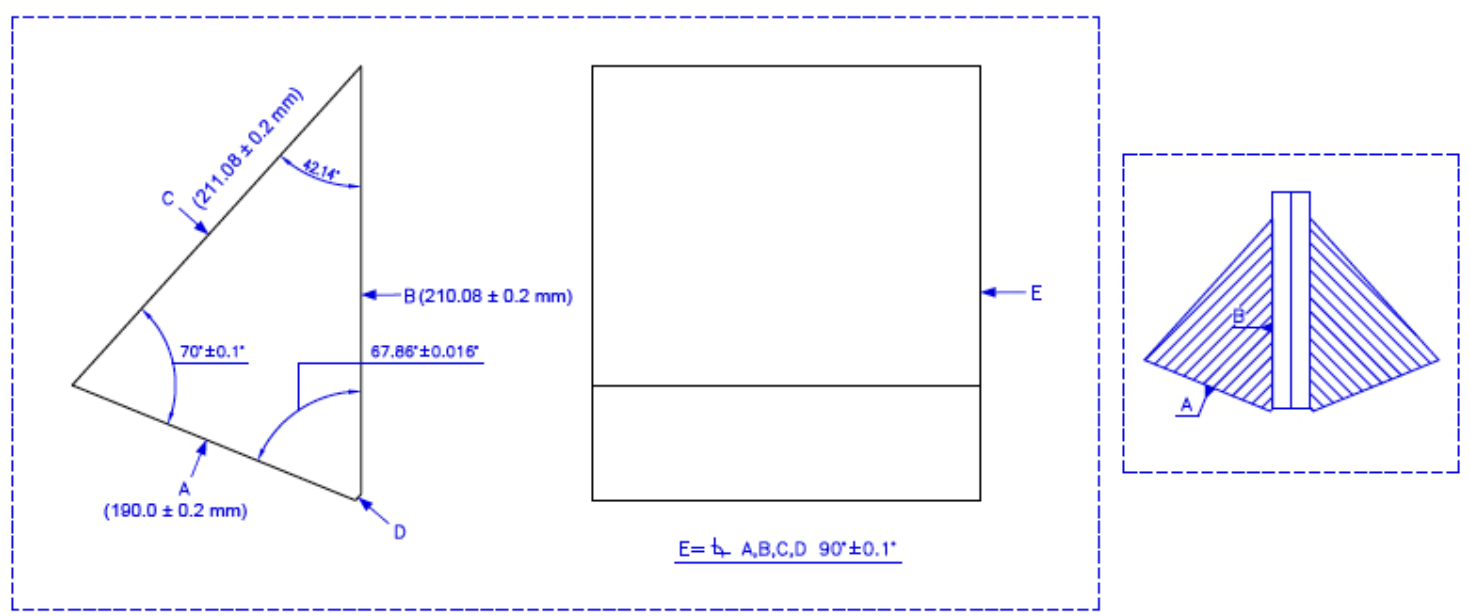

Figure 9: HR prisms drawing map

Proc. of SPIE Vol. 9912 99124D-5 
Table 2: Main parameters that characterizes the VPHs for the LR, MR, and HR modes respectively

\begin{tabular}{|c|c|c|c|c|c|c|c|}
\hline \multicolumn{8}{|c|}{ Optical Parameters } \\
\hline VPHs & ID & $\lambda \mathrm{c}(\AA)$ & $\lambda_{1}-\lambda_{2}(\AA)$ & $\sigma\left(\operatorname{lin} \mathrm{mm}^{-1}\right)$ & AOE & Bragg & $\mathbf{R}_{\text {FWHM }}$ \\
\hline \multirow{6}{*}{ LR } & LR-U & 4051 & $3653-4386$ & $2761 \pm 3$ & $34.0^{\circ}$ & $26.12^{\circ}$ & 6028 \\
\hline & LR-B & 4800 & $4332-5196$ & $2330 \pm 3$ & $34.0^{\circ}$ & $26.12^{\circ}$ & 6059 \\
\hline & LR-V & 5695 & $5143-6164$ & $1964 \pm 3$ & $34.0^{\circ}$ & $26.12^{\circ}$ & 6080 \\
\hline & LR-R & 6747 & $6094-7300$ & $1658 \pm 3$ & $34.0^{\circ}$ & $26.12^{\circ}$ & 6099 \\
\hline & LR-I & 7991 & $7220-8646$ & $1400 \pm 3$ & $34.0^{\circ}$ & $26.12^{\circ}$ & 6110 \\
\hline & LR-Z & 8900 & $8043-9630$ & $1257 \pm 3$ & $34.0^{\circ}$ & $26.12^{\circ}$ & 6117 \\
\hline \multirow{10}{*}{ MR } & MR-U & 4104 & $3917-4277$ & $4942 \pm 3$ & --- & $52.98^{\circ}$ & 12602 \\
\hline & MR-UB & 4431 & $4225-4621$ & $4551 \pm 3$ & --- & $52.55^{\circ}$ & 12370 \\
\hline & MR-B & 4814 & $4586-5024$ & $4167 \pm 3$ & --- & $52.16^{\circ}$ & 12178 \\
\hline & MR-G & 5213 & $4963-5443$ & $3832 \pm 3$ & $87.26^{\circ}$ & $51.86^{\circ}$ & 12035 \\
\hline & MR-V & 5667 & $5393-5919$ & $3513 \pm 3$ & $84.44^{\circ}$ & $51.60^{\circ}$ & 11916 \\
\hline & MR-VR & 6170 & $5869-6447$ & $3217 \pm 3$ & $82.93^{\circ}$ & $51.39^{\circ}$ & 11825 \\
\hline & MR-R & 6563 & $6241-6859$ & $3018 \pm 3$ & $82.02^{\circ}$ & $51.24^{\circ}$ & 11768 \\
\hline & MR-RI & 7115 & $6764-7437$ & $2778 \pm 3$ & $81.20^{\circ}$ & $51.09^{\circ}$ & 11707 \\
\hline & MR-I & 7767 & $7382-8120$ & $2540 \pm 3$ & $80.50^{\circ}$ & $50.95^{\circ}$ & 11654 \\
\hline & MR-Z & 9262 & $8800-9686$ & $2123 \pm 3$ & $79.48^{\circ}$ & $50.73^{\circ}$ & 11638 \\
\hline \multirow{2}{*}{ HR } & HR-R & 6646 & $6445-6837$ & $3592 \pm 3$ & --- & $70.12^{\circ}$ & 18700 \\
\hline & HR-I & 8634 & $8372-8882$ & $2761 \pm 3$ & --- & $69.73^{\circ}$ & 18701 \\
\hline
\end{tabular}

\section{TESTS}

At the time when this paper was submitted all MEGARA gratings (and any prism) were not supplied to our facilities, therefore the tests described in this section are carried out with the gratings available, although the set up is the same for all of them.

\subsection{Line density}

The capability to produce the dispersion of the light by the VPH grating comes from the grooves engraved in the gelatin sandwiched between the two glass substrates. The grooves pattern is carried out by means of an engraving laser technique. This process is decisive and is very important that the line density be that require for the VPH because this has a deep impact in the final resolution of the grating and therefore in the optimal running of the spectrograph.

For this test we will use a green laser with a wavelength at $532 \mathrm{~nm}$, an autocollimator, a goniometer and the VPH previously placed in a mount. The set-up configuration can be seen in Figure 10. The VPH is placed at the top of a goniometer and perpendicular to the laser beam that passes directly through it. We will determine the position 1 (Pos. 1 in the figure) when the return of the laser beam is imaged in the autocollimator eyepiece (the light that is reflected in the VPH surface and goes back through the same path to the autocollimator). 

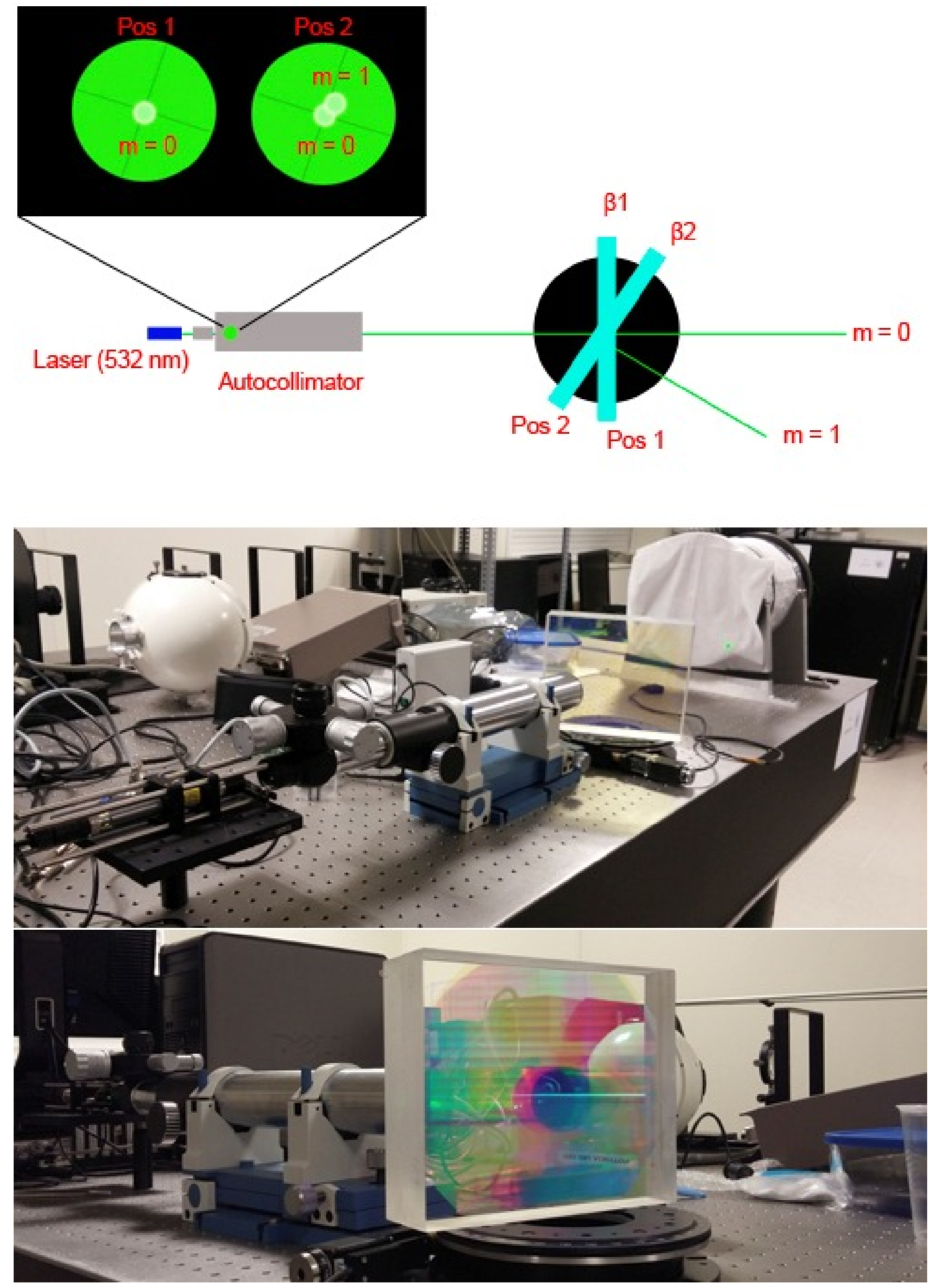

Figure 10: Setup designed for deriving the grating line density 
At position Pos. 1 the angle of reference is measured $(\beta 1)$, later on the goniometer is rotated until the diffracting order $m=1$ is produced and its reflected beam is seen at the autocollimator eyepice, obtaining the position Pos. 2 and the angle $\beta 2$. Using the Snell diffraction law when the laser pass through the air to the inner side of the VPH (that is, through the gelatin) we have:

$$
n_{1} \sin \beta=n_{2} \sin \alpha
$$

Equation 1

where $n_{l}$ is the air refraction index $\left(n_{1}=1\right)$ and $\beta=\beta 2-\beta 1$, and $n_{2}$ is the gelatin refractive index. Using the diffraction grating in Bragg condition we have:

$$
2 \sin \alpha=\sigma \lambda
$$

Equation 2

And therefore the line density as:

$$
\sigma=\frac{2 \sin \alpha}{\lambda}
$$

Equation 3

\section{Total internal reflection}

One solution for increasing the spectral dispersion, and therefore the power resolution, of a grating without modifying any of their structural and optical parameters (i.e., the number of grooves, the spectral order, etc.), is increasing the angle of incidence $\beta$ as Equation 4 shows:

$$
\frac{d \beta}{d \lambda}=\frac{m \sigma}{\cos \beta}
$$

Equation 4

In the case of MEGARA, this is performed thanks to a single prism glued to the flat VPH window that produces a total reflection in one of its internal faces, driving the light beam toward the flat window with high incidence angles.

As the final configuration of the pupil elements for MR an HR are composed by a VPH sandwiched between two single prisms (see Figure 8 and Figure 9), those gratings with high Bragg angles would suffer a TIR in the returning light beam, which would not allow it coming out from the VPH unless the prism is joined. This issue affects those VPHs not obeying the following condition:

$$
\beta_{\text {Bragg }} \leq 51.94^{\circ}
$$

Equation 5

For avoiding this issue, the test is configured with the help of a right-angle prism that will let using the same laser for all gratings. Table 3 shows the gratings that will be used with the right-angle prism and the AOE respect to the prism face. The prism must be in contact with the VPH flat surface on one of its legs. Three configurations will be used:

1. Incident light beam toward the VPH flat window, see Figure 11 top.

2. Incident light beam toward the prism face at $45^{\circ}$, see Figure 11 middle.

3. Incident light beam toward the prism face at $90^{\circ}$, see Figure 11 bottom. 
Table 3: AOI angles for using the $532 \mathrm{~nm}$ laser with all the VPHs, and with the help of a right-angle prism in those necessary situations. Angles are measured from the normal to the VPH surface

\begin{tabular}{|c|c|c|c|c|c|}
\hline & & & \multicolumn{3}{|c|}{ Setup Entrance Angle } \\
\hline VPHs & Setup & $\sigma\left(\operatorname{lin} \mathrm{mm}^{-1}\right)$ & No prism & $\begin{array}{c}\text { Prism entering } \\
\text { legs }\end{array}$ & $\begin{array}{l}\text { Prism entering } \\
\text { hypotenuse }\end{array}$ \\
\hline \multirow[t]{6}{*}{ LR } & LR-U & $2761 \pm 3$ & $47.26^{\circ}$ & -- & --- \\
\hline & LR-B & $2330 \pm 3$ & $38.10^{\circ}$ & --- & --- \\
\hline & LR-V & $1694 \pm 3$ & $26.78^{\circ}$ & --- & --- \\
\hline & LR-R & $1658 \pm 3$ & $26.17^{\circ}$ & --- & --- \\
\hline & LR-I & $1400 \pm 3$ & $21.86^{\circ}$ & --- & --- \\
\hline & LR-Z & $1257 \pm 3$ & $19.53^{\circ}$ & --- & --- \\
\hline \multirow{10}{*}{ MR } & MR-U & $4942 \pm 3$ & --- & --- & $40.44^{\circ}$ \\
\hline & MR-UB & $4551 \pm 3$ & --- & $61.14^{\circ}$ & $35.72^{\circ}$ \\
\hline & MR-B & $4167 \pm 3$ & --- & $51.38^{\circ}$ & --- \\
\hline & MR-G & $3832 \pm 3$ & --- & $43.91^{\circ}$ & --- \\
\hline & MR-V & $3513 \pm 3$ & --- & $37.35^{\circ}$ & --- \\
\hline & MR-VR & $3217 \pm 3$ & --- & $31.56^{\circ}$ & --- \\
\hline & MR-R & $3018 \pm 3$ & --- & $27.83^{\circ}$ & --- \\
\hline & MR-RI & $2778 \pm 3$ & $47.64^{\circ}$ & $23.38^{\circ}$ & --- \\
\hline & MR-I & $2540 \pm 3$ & $42.50^{\circ}$ & --- & --- \\
\hline & MR-Z & $2123 \pm 3$ & $34.38^{\circ}$ & --- & --- \\
\hline \multirow{2}{*}{ HR } & HR-R & $3592 \pm 3$ & --- & $38.84^{\circ}$ & --- \\
\hline & HR-I & $2761 \pm 3$ & $47.26^{\circ}$ & --- & --- \\
\hline
\end{tabular}

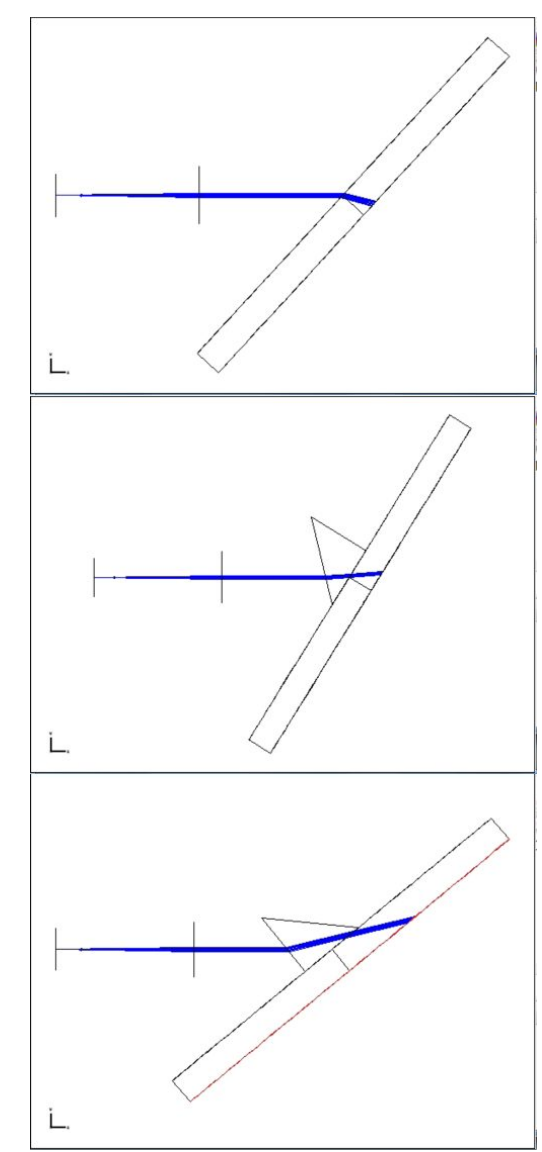

Figure 11: Layout of the three different configurations, depending on the VPH used, for performing the line density test 


\subsection{Efficiency}

For obtaining the efficiency curve of each grating we make use of an Oriel CornerStone 260 monochromator that is able to cover all MEGARA spectral range with a resolution of $0.1 \mathrm{~nm}$. The setup is that shown in Figure 12. We place an integrating sphere provided with a calibrated Keithley photodiode. We obtained a photometric run covering all the MAGARA spectral range (our reference and calibrated curve, TransRef), see Figure 12 top. Then each grating is placed perpendicular to the monochromator optical axis collecting all the light behind it with an integrating sphere and forming an angle of 34 degrees relative the optical axis (i.e. the AOI at the instrument). We made a new scan measuring again the transmission in order zero, registering all the flux through the grating (TransGrating) not diffracted but that one in the spectral range of the grating, which is "gone" towards the diffraction order 1. That is possible due to the high efficiency that offers a VPH, because the flux in other orders is nearly null, see Figure 12 bottom. Therefore the efficiency is obtained as:

$$
\varepsilon=1-\frac{\text { TransGrating }}{\text { Trans } \operatorname{Re} f}
$$
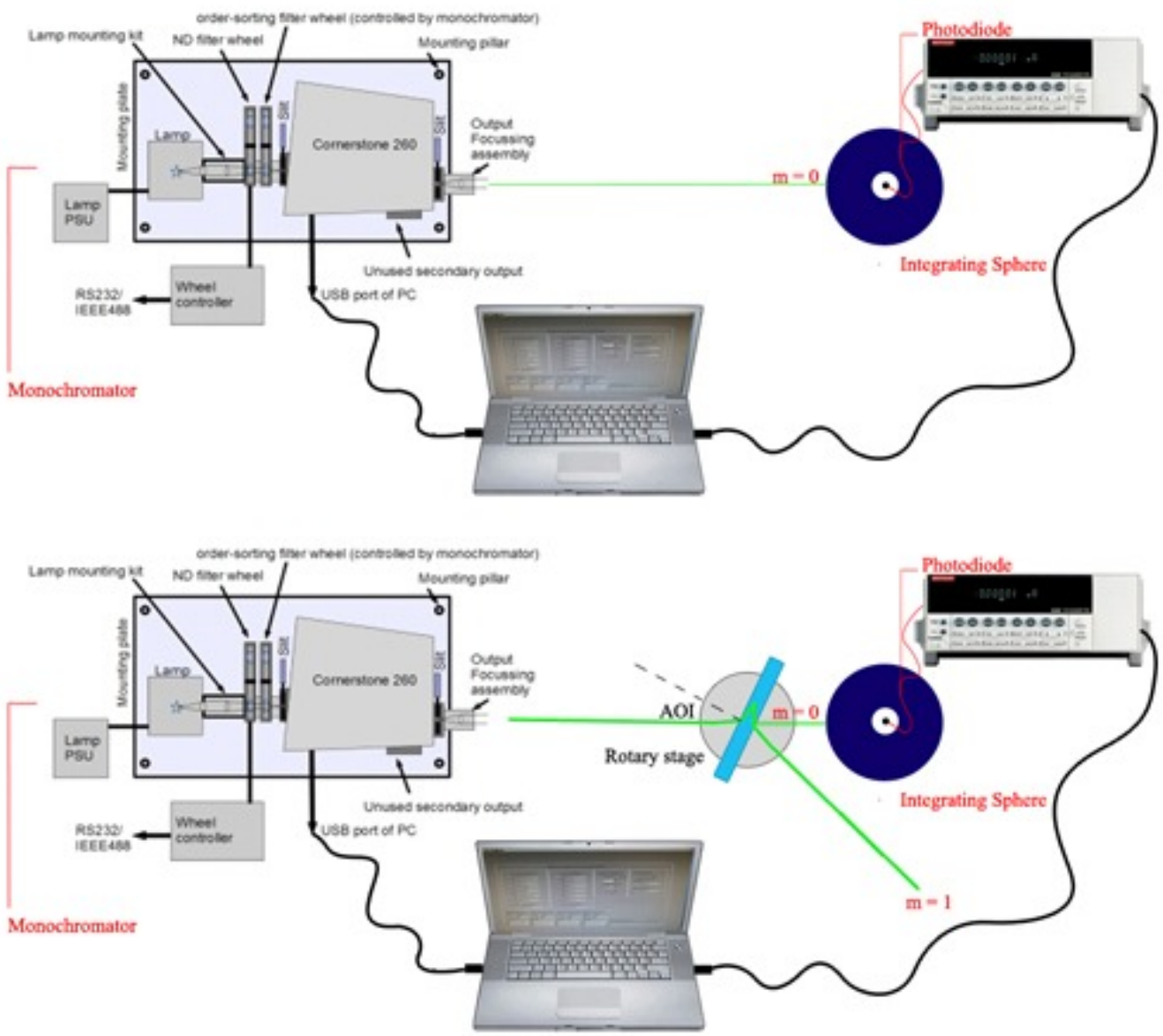

Figure 12: Setup designed for obtaining the efficiency curve of each grating 


\section{RESULTS}

As mentioned previously not all the gratings were available at the moment of writing this proceeding, therefore we put the results for $4 \mathrm{LR}$ gratings and $1 \mathrm{HR}$. The following set of tables show the values obtained in order to derive the line density:

Table 4: Line desnsity results for gratings LR-V, LR-R, LR-I, LR-Z and HR-I respectively

\begin{tabular}{|c|c|c|}
\hline Grating LR-V & $\mathbf{m}=1$ & $m=-1$ \\
\hline$\beta_{1}$ & \multicolumn{2}{|c|}{$45^{\prime}$} \\
\hline $\boldsymbol{\beta}_{2}$ & $32^{\circ} 15^{\prime}$ & $329^{\circ} 20^{\prime}$ \\
\hline$\beta$ & $31.50^{\circ}$ & $31.42^{\circ}$ \\
\hline$\sigma\left(\operatorname{lin} \mathrm{mm}^{-1}\right)$ & 1964 & 1960 \\
\hline$<\sigma>\left(\operatorname{lin} \mathrm{mm}^{-1}\right)$ & \multicolumn{2}{|c|}{$1962 \pm 3$} \\
\hline
\end{tabular}

\begin{tabular}{|l|r|r|}
\hline Grating LR-R & $\mathbf{m}=\mathbf{1}$ & $\mathbf{m}=\mathbf{- 1}$ \\
\hline $\boldsymbol{\beta}_{\mathbf{1}}$ & \multicolumn{2}{|c|}{$5^{\circ} 20^{\prime}$} \\
\hline $\boldsymbol{\beta}_{\mathbf{2}}$ & $31^{\circ} 25^{\prime} 12^{\prime \prime}$ & $339^{\circ} 10^{\prime} 15^{\prime \prime}$ \\
\hline $\boldsymbol{\beta}$ & $26.09^{\circ}$ & $26.16^{\circ}$ \\
\hline $\boldsymbol{\sigma}\left(\right.$ lin $\left.\mathbf{~ m m}^{-1}\right)$ & 1653 & 1657 \\
\hline$<\boldsymbol{\sigma}>\left(\right.$ lin $\left.\mathbf{~ m m}^{-1}\right)$ & \multicolumn{2}{|c|}{$1655 \pm 3$} \\
\hline
\end{tabular}

\begin{tabular}{|l|r|r|r|r|}
\hline Grating LR-I & \multicolumn{1}{|c|}{$\mathbf{m}=\mathbf{2}$} & $\mathbf{m}=\mathbf{1}$ & \multicolumn{1}{c|}{$\mathbf{m}=\mathbf{- 1}$} & \multicolumn{1}{c|}{$\mathbf{m}=\mathbf{- 2}$} \\
\hline $\boldsymbol{\beta}_{\mathbf{1}}$ & \multicolumn{5}{|c|}{$358^{\circ} 45^{\prime}$} \\
\hline $\boldsymbol{\beta}_{\mathbf{2}}$ & $46^{\circ} 50^{\prime} 9^{\prime \prime}$ & $20^{\circ} 40^{\prime} 6^{\prime \prime}$ & $336^{\circ} 55^{\prime} 6^{\prime \prime}$ & $310^{\circ} 40^{\prime}$ \\
\hline $\boldsymbol{\beta}$ & $48.09^{\circ}$ & $21.92^{\circ}$ & $21.83^{\circ}$ & $48.08^{\circ}$ \\
\hline $\boldsymbol{\sigma}\left(\operatorname{lin} \mathbf{~ m m}^{-1}\right)$ & 1399 & 1403 & 1398 & 1399 \\
\hline$<\boldsymbol{\sigma}>\left(\operatorname{lin} \mathbf{~ m m}^{-1}\right)$ & \multicolumn{5}{|c}{} \\
\hline
\end{tabular}

\begin{tabular}{|l|r|r|r|r|}
\hline Grating LR-Z & \multicolumn{1}{|c|}{$\mathbf{m}=\mathbf{2}$} & $\mathbf{m}=\mathbf{1}$ & \multicolumn{1}{c|}{$\mathbf{m}=\mathbf{- 1}$} & \multicolumn{1}{c|}{$\mathbf{m}=\mathbf{- 2}$} \\
\hline $\boldsymbol{\beta}_{\mathbf{1}}$ & \multicolumn{5}{|c|}{$50^{\circ} 15^{\prime}$} \\
\hline $\boldsymbol{\beta}_{\mathbf{2}}$ & $42^{\mathbf{o}} 45^{\prime}$ & $20^{\circ} 20^{\prime}$ & $341^{\circ} 20^{\prime}$ & $318^{\circ} 55^{\prime}$ \\
\hline $\boldsymbol{\beta}$ & $41.91^{\circ}$ & $19.50^{\circ}$ & $19.50^{\circ}$ & $41.92^{\circ}$ \\
\hline $\boldsymbol{\sigma}\left(\operatorname{lin} \mathbf{~ m m}^{-1}\right)$ & 1256 & 1255 & 1255 & 1256 \\
\hline$<\boldsymbol{\sigma}>\left(\operatorname{lin} \mathbf{~ m m}^{-1}\right)$ & & \multicolumn{2}{|c|}{$1256 \pm 3$} & \\
\hline
\end{tabular}

\begin{tabular}{|c|c|c|}
\hline Grating HR-I & $\mathbf{m}=1$ & $m=-1$ \\
\hline $\boldsymbol{\beta}_{1}$ & \multicolumn{2}{|c|}{$10^{\prime}$} \\
\hline $\boldsymbol{\beta}_{2}$ & $47^{\circ} 20^{\prime}$ & $312^{\circ} 50^{\prime}$ \\
\hline$\beta$ & $47.16^{\circ}$ & $47.33^{\circ}$ \\
\hline$\sigma\left(\operatorname{lin} \mathrm{mm}^{-1}\right)$ & 2757 & 2764 \\
\hline$<\sigma>\left(\right.$ lin $\left.\mathrm{mm}^{-1}\right)$ & \multicolumn{2}{|c|}{$2760 \pm 3$} \\
\hline
\end{tabular}


And the following set of frames (Figure 13) show the efficiency curves derived for all the low-resolution gratings, with the exception of the HR one that has not been measured yet.
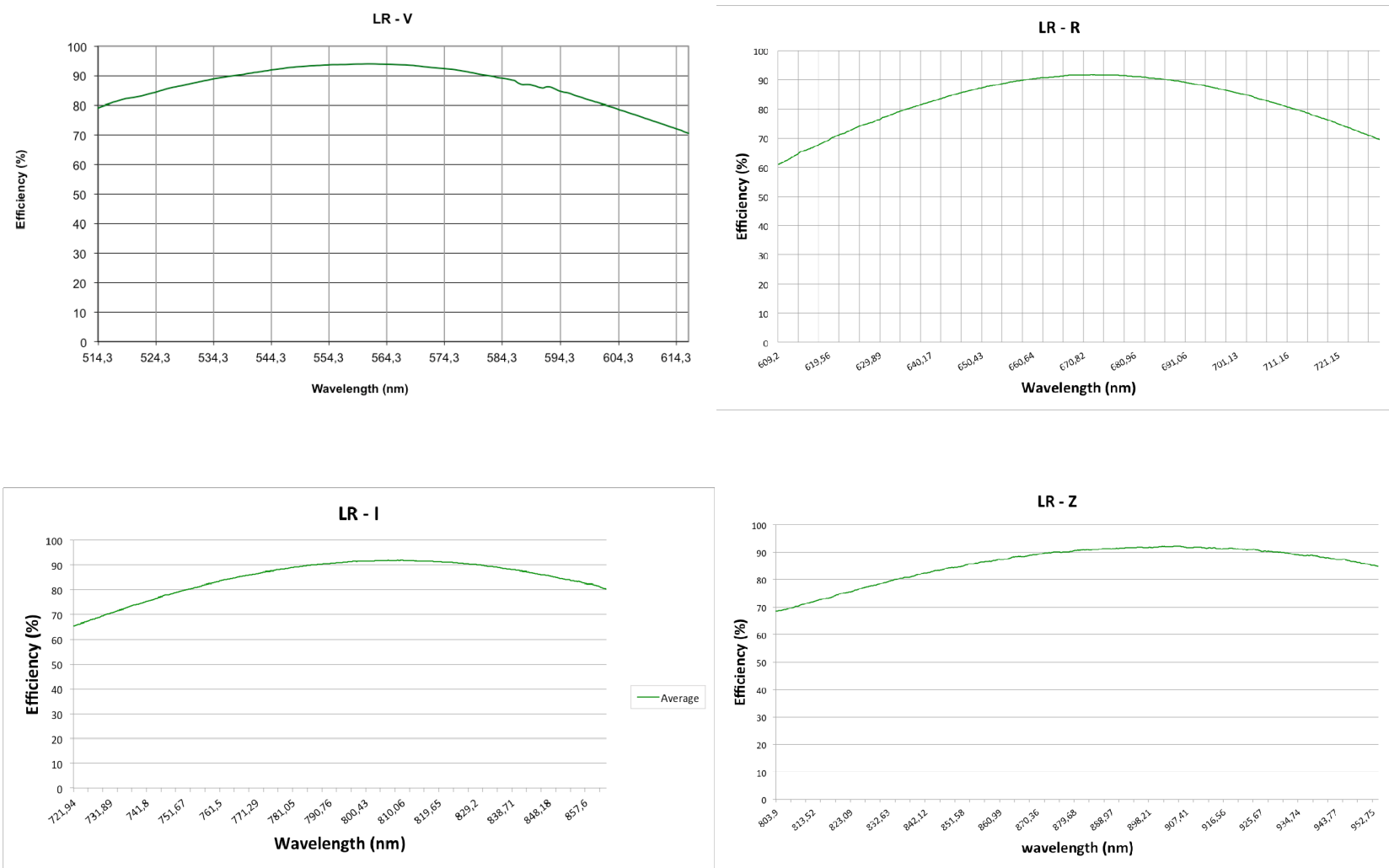

Figure 13: Efficiency curves for gratings LR-V, LR-R, LR-I and LR-Z respectively

\section{SUMMARY}

We have described the setup developed to validate and check some important requirements for MEGARA pupil elements, as the line density and the efficiency curve of each dispersive element. We can infer from the results that the gratings available for the instrument are under requirements

\section{REFERENCES}

[1] Gil de Paz, A., et al., "MEGARA: The future optical IFU and MOS for the 10.4 GTC telescope," Proc. SPIE 8846, Ground-based and Airborne Instrumentation for Astronomy IV, 84464Q (2012).

[2] García-Vargas, M.L., et al., "MEGARA optical design: the new integral field unit and multi-object spectrograph for the GTC 10m telescope," Proc. SPIE 8550, Ground-based and Airborne Instrumentation for Astronomy IV, 85501C (2012). 